\title{
The role of animal models in advancing amyloid-beta immunotherapy to the clinic
}

\author{
Dora Games* and Peter Seubert
}

\begin{abstract}
The amyloid-beta (A $\beta$ ) hypothesis of Alzheimer's disease (AD) causality is now well into its third decade and is finally entering a phase of rigorous clinical testing in numerous late stage clinical trials. The use of $A \beta$-based animal models of AD has been essential to the discovery and/or preclinical validation of many of these therapeutic approaches. While several neuropathologically based results from preclinical studies have translated nicely into AD patients, the full clinical value of $A \beta$-directed therapies awaits results from trials now in progress.
\end{abstract}

The available treatment options for Alzheimer's disease (AD), the most common chronic, progressive (and inevitably fatal) dementing neurological disease, have been disappointing in both their paucity and effectiveness as compared to other areas of medical intervention such as cardiovascular disease and oncology. The AD therapeutic picture is hopefully on the verge of a major positive change, as numerous clinical trials are now testing agents specifically designed to target hypothesized etiologic agents of $\mathrm{AD}$, as opposed to the current class of approved therapies that offer only modest and transient symptomatic relief. One such hypothesis-driven approach is amyloid-beta $(\mathrm{A} \beta)$ immunotherapy, which is under investigation in over a dozen clinical trials by a multitude of different sponsors, including several late stage phase 3 studies. The results of these trials are eagerly anticipated as the underlying rationale directly tests the $A \beta$ hypothesis and is supported by extraordinarily compelling preclinical data. Both the scientific origin and evolution of this approach were intimately interwoven with the successful exploitation of human genetic findings that led

*Correspondence: Dora.Games@neotopebio.com

Janssen Alzheimer Immunotherapy Research and Development, LLC, 700 Gateway Blvd, South San Francisco, CA 94080, USA to the development of transgenic animal models of this progressive central nervous system disease.

Over a century ago, Dr Alois Alzheimer described the neuropathological brain lesions that, to this day, are essential in the post-mortem confirmation of AD diagnosis, namely amyloid plaques and neurofibrillary tangles. These lesions are composed primarily of the $A \beta$ peptide and microtubule-associated tau protein, respectively, leaving the field to question whether one, both or neither of these proteins are causative of $A D$. $A \beta$ is a peptide prone to self-aggregation and fibril formation leading to formation of extracellular plaques. The identification of genetic mutations - linked to autosomal dominant forms of $A D$ in the amyloid precursor protein, from which $A \beta$ is derived - not only bolstered the amyloid hypothesis but allowed for the generation of transgenic mice that recapitulated many (but not all) human AD-like pathologies, including plaque formation, loss of synapses, neuroinflammation, cognitive impairments and neuritic dystrophy. A major significance of these findings is that therapies can now be tested for efficacy against amyloidrelated pathologies in a tangible context of disease. After many years of using human pathology to determine relevancy of animal models to disease, the converse challenge emerged: would alterations in pathological cascades seen in animal models accurately predict human response to therapeutic interventions?

$A \beta$ immunotherapy is a deceptively simple idea; expose the $\mathrm{AD}$ diseased brain to anti-A $\beta$ antibodies, either by 'active' immunization (injection of an $A \beta$-containing immunogen to stimulate a host response) or 'passive' immunization (administration of a recombinant monoclonal antibody) to stimulate the body's defenses to neutralize and clear $A \beta$ as it would other invasive entities. The first results with active $A \beta$ immunotherapy [1] were remarkable in the extent to which the amyloid-associated pathologies could be reduced in a transgenic model. Importantly, not just the amyloid plaques were cleared, but synapses were preserved, neuritic dystrophy was reduced and neuro-inflammation subsided. These results were rapidly replicated and expanded upon in labs around the world and now behavioral deficits and neuronal loss [2] can be included in the list of preclinical in vivo 
endpoints improved by $\mathrm{A} \beta$ immunotherapy. The ultimate validation of their translational accuracy is the purview of ongoing late stage clinical trials, but several encouraging results have been reported. The first demonstration of treatment-associated plaque reduction in $\mathrm{AD}$ brain was identified upon post-mortem evaluation of an actively immunized patient [3]. Reduction in neuritic dystrophy was also observed, and the presence of activated microglia with internalized $A \beta$ indicated at least one downstream cellular mechanism was similarly invoked in both human and transgenic mouse brains. In recent subsequent studies, the reduction of tau-positive neuropil threads (plaque-associated but not somatic tangles) has also been reported in actively immunized AD patients (James Nicholl, personal communication). Encouragingly, passive immunotherapy with bapineuzumab has been also shown to reduce amyloid burden, as visualized by $\mathrm{PET} / \mathrm{PiB}$ methodology, in living $\mathrm{AD}$ patients [4]. Interestingly, the divergence of $\mathrm{PiB}$ burdens between control and bapineuzumab-treated patients showed a similar time course of separation to that of the clinical measures in patients who completed (completers) all doses in a Phase 2 Bapineuzumab trial [5]. Success in the ongoing phase 3 trials of bapineuzumab would not only be a tremendous validation of preclinical modeling of a progressive neurological disease, but would represent a new era in hypothesis-driven, disease-modifying therapy for this poorly served patient population.

It is difficult to contemplate how this therapeutic approach could have advanced so far in the absence of a suitable animal model of the disease. The therapy itself involves the interplay of numerous in vivo systems impossible to adequately model otherwise. In the case of active therapy, the therapeutic response begins with host recognition of the $A \beta$-derived immunogen and subsequent mounting of an antibody-raising immune response. In both active and passive therapies, the antibodies likely cross the blood-brain barrier (approximately $0.1 \%$ of serum IgG levels are found in the central nervous system [6]), bind to the target and trigger neutralization, clearance and/or removal of pathogenic $A \beta$ species. Acute behavioral improvements (due presumably to neutralizing the effects of brain interstitial synapto-toxic $A \beta$ oligomers) and progressive reductions in $A \beta$-associated neuropathologies are seen in preclinical rodent and non-human models following administration of anti- $\mathrm{A} \beta$ antibodies.
Whether these results will translate effectively to the complex clinical outcomes awaits the completion of human testing. The compelling and extensive replication of numerous convergent efficacy measures by independent investigators and confirmation of activity in humans continue to place $A \beta$ immunotherapy in the forefront of potential new treatments for $\mathrm{AD}$. The use of transgenic animal models was essential in this case, and these models can be expected to continue to elucidate disease mechanisms and thereby enable effective approaches to treat, and eventually prevent, the tragic course of AD.

\section{Abbreviations}

$A \beta=$ amyloid-beta; $A D=$ Alzheimer's disease.

\section{Competing interests}

The authors are dual employees of Janssen Alzheimer Immunotherapy Research and Development, LLC and Neotope Biosciences, a division of Elan Pharmaceuticals.

Published: 29 July 2010

\section{References}

1. Schenk D, Barbour R, Dunn W, Gordon G, Grajeda H, Guido T, Hu K, Huang J, Johnson-Wood K, Khan K, Kholodenko D, Lee M, Liao Z, Lieberburg I, Motter R, Mutter L, Soriano F, Shopp G, Vasquez N, Vandevert C, Walker S, Wogulis M, Yednock T, Games D, Seubert P: Immunization with amyloid-beta attenuates Alzheimer-disease-like pathology in the PDAPP mouse. Nature 1999, 400:173-177.

2. Wilcock DM, Gharkholonarehe N, Van Nostrand WE, Davis J, Vitek MP, Colton CA: Amyloid reduction by amyloid-beta vaccination also reduces mouse tau pathology and protects from neuron loss in two mouse models of Alzheimer's disease. J Neurosci 2009, 29:7957-7965.

3. Nicoll JA, Wilkinson D, Holmes C, Steart P, Markham H, Weller RO: Neuropathology of human Alzheimer disease after immunization with amyloid-beta peptide: a case report. Nat Med 2003, 9:448-452.

4. Rinne JO, Brooks DJ, Rossor MN, Fox NC, Bullock R, KlunkWE, Mathis CA, Blennow K, Barakos J, Okello AA, Rodriguez Martinez de Liano S, Liu E, Koller M, Gregg KM, Schenk D, Black R, Grundman M: 11C-PiB PET assessment of change in fibrillar amyloid-beta load in patients with Alzheimer's disease treated with bapineuzumab: a phase 2, double-blind, placebo-controlled, ascending-dose study. Lancet Neurol 2010, 9:363-372.

5. Salloway S, Sperling R, Gilman S, Fox NC, Blennow K, Raskind M, Sabbagh M, Honig LS, Doody R, van Dyck CH, Mulnard R, Barakos J, Gregg KM, Liu E, Lieberburg I, Schenk D, Black R, Grundman M; Bapineuzumab 201 Clinical Trial Investigators: A phase 2 multiple ascending dose trial of bapineuzumab in mild to moderate Alzheimer disease. Neurology 2009, 73:2061-2070.

6. Banks WA, Terrell B, Farr SA, Robinson SM, Nonaka N, Morley JE: Passage of amyloid beta protein antibody across the blood-brain barrier in a mouse model of Alzheimer's disease. Peptides 2002, 23:2223-2226.

doi:10.1186/alzrt46

Cite this article as: Games D, Seubert P: The role of animal models in advancing amyloid-beta immunotherapy to the clinic. Alzheimer's Research \& Therapy 2010, 2:22. 